\title{
Optimization of Inertial Micropower Generators for Human Walking Motion
}

\author{
Thomas von Büren, Paul D. Mitcheson, Tim C. Green, Eric M. Yeatman, Andrew S. Holmes, and Gerhard Tröster
}

\begin{abstract}
Micropower generators, which have applications in distributed sensing, have previously been classified into architectures and analyzed for sinusoidal driving motions. However, under many practical operating conditions, the driving motion will not be sinusoidal. In this paper, we present a comparison of the simulated performance of optimized configurations of the different architectures using measured acceleration data from walking motion gathered from human subjects. The sensitivity of generator performance to variations in generator parameters is investigated, with a $20 \%$ change in generator parameters causing between a $3 \%$ and $80 \%$ drop in generator power output, depending upon generator architecture and operating condition. Based on the results of this investigation, microgenerator design guidelines are provided. The Coulomb-force parametric generator is the recommended architecture for generators with internal displacement amplitude limits of less than $\sim 0.5 \mathrm{~mm}$ and the velocity-damped resonant generator is the recommended architecture when the internal displacement amplitude can exceed $\sim 0.5 \mathrm{~mm}$, depending upon the exact operating conditions. Maximum power densities for human powered motion vary between 8.7 and $2100 \mu \mathrm{W} / \mathrm{cm}^{3}$, depending upon generator size and the location of the body on which it is mounted.
\end{abstract}

Index Terms-Micropower generator, micropower supply, vibration-to-electric energy conversion.

\section{INTRODUCTION}

$\mathbf{T}$ RADITIONALLY, healthcare has concentrated upon the short-term treatment of life threatening problems in hospital beds rather than long term monitoring and prevention of chronic illness [1]. However, many chronically ill patients could have a significant increase in their quality of life and life expectancy if certain biological signs could be continually monitored during their daily lives. For example, continually monitoring blood pressure in patients with hypertension can significantly increase medication compliance leading to a significant reduction in blood pressure [2]. Real-time processing of electrocardiograph traces can be very effective at revealing the early stages of heart disease [3], [4].

\footnotetext{
Manuscript received July 9, 2004; revised August 25, 2004. This research was supported in part by ETH Zurich under the Polyproject "Wearable Computing: Technology and Applications," in part by the Imperial College London under an EPSRC Portfolio award, and in part by the ORESTEIA project which is part of the DC Initiative (EU Framework V). The associate editor coordinating the review of this paper and approving it for publication was Dr. Subhas Mukhopadhyay.

T. von Büren and G. Tröster are with the Electronics Laboratory, Department of Information Technology and Electrical Engineering, Swiss Federal Institute of Technology (ETH) Zurich, CH-8092 Zurich, Switzerland (e-mail: tvonbuer@ife.ee.ethz.ch; troester@ife.ee.ethz.ch).

P. D. Mitcheson, T. C. Green, E. M. Yeatman, and A. S. Holmes are with the Department of Electrical and Electronic Engineering, Imperial College London, London SW7 2AZ, U.K. (e-mail: paul.mitcheson@ imperial.ac.uk; t.green@imperial.ac.uk; $\quad$ e.yeatman@imperial.ac.uk; a.holmes@imperial.ac.uk).

Digital Object Identifier 10.1109/JSEN.2005.853595
}

Medical sensors and sensor networks [5] capable of performing these tasks will only significantly increase quality of life if they are unobtrusive to the patient. Some of the sensors are implantable and consequently regular maintenance should be avoided, so frequent replacement of the power source is undesirable. Additionally, such body-worn autonomous sensor systems have many applications in the field of context awareness [6] and the Ubiquitous Computing paradigm [7]. Context awareness can be described as the ability of a computer system to detect various aspects of the user's situation (such as location, activity, vital signs, and state of the environment) and to adapt its behavior accordingly [8], [9]. Many such systems rely on distributed sensor networks. As with medical systems, very little or no maintenance is a prerequisite for these applications.

A possible alternative to the use of finite internal energy sources (i.e., batteries) in these applications is that of scavenging energy from the environment of the device. Various energy-scavenging techniques which could be used to power medical and other sensors have previously been reported, including ones utilizing thermal gradients [10], [11], solar energy [12], [13], and electromagnetic waves [14]. However, powering devices from the motion of the devices themselves has attracted the most interest [15]-[23]. A comprehensive overview of power sources for wearable electronics is presented in [24].

Two types of motion-powered microgenerators have been reported, these being force-driven generators and inertial (or vibration-driven) generators. In force-driven generators, the mechanical energy is converted by the direct application of force. An example of this type of generator (making use of foot to ground impact) was presented in [15]. In inertial generators the energy is coupled into the system by the inertia of a moving mass within the generator frame [16]-[23]. In this work, only inertial generators are considered.

Inertial microgenerators are mixed electromechanical systems, in which mechanical motion is used to generate electrical energy through the use of an electric or magnetic field, or a piezoelectric material. However, it is possible to model ideal implementations of these generators as a mechanical system only, i.e., a second-order mass-spring system, where the electrical energy generated is represented as the energy dissipated in a mechanical damper. The operating principle of inertial microgenerators is shown in Fig. 1. The inertia of a proof mass $m$, which is suspended on a spring suspension with spring constant $k$, causes the mass to move relative to the generator frame with relative displacement $z(t)$ when the frame, with displacement $y(t)$, experiences acceleration. The maximum and minimum values of $z(t)$ are $\pm Z_{l}$, imposed by the finite size of the generator. Energy is converted when work is done against 


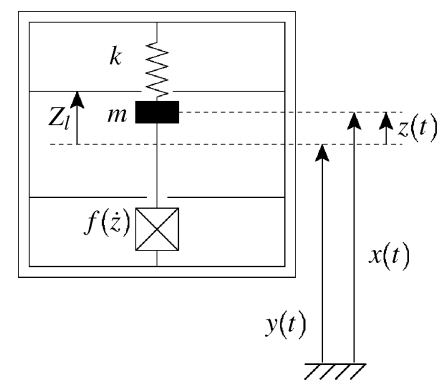

Fig. 1. Generic model of inertial generator.

a damping force $f(\dot{z})$ which opposes the relative motion of the proof mass.

The chosen energy-conversion principle determines the velocity-force characteristic of that damping force, and the characteristic of this force determines the achievable power density.

Inertial microgenerators have been classified into three main architectures in [25], depending upon the overall operating strategy of the generator and the velocity-force characteristic of the energy-conversion mechanism. Fig. 2 shows the operation of the generators for a sinusoidal displacement input, $y(t)$, with amplitude $1 \mathrm{~mm}$ and frequency $10 \mathrm{~Hz}$, showing the relative mass-frame velocity $d z / d t$, the damping force $f(\dot{z})$, and the relative displacement $z(t)$. The architectures are briefly summarized below.

1) Velocity-damped resonant generator (VDRG). In this device, usually implemented with electromagnetics or piezoelectrics, the damping force is proportional to the relative velocity between the proof mass and the generator frame (i.e., $f=-D \cdot \dot{z}$, where $D$ is a constant coefficient, called the damping coefficient) [16]-[21]. The dashed lines in Fig. 2 show the velocity, damping, and displacement for a VDRG for $m=1 \mathrm{~g}, k=80 \mathrm{~N} / \mathrm{m}$ and $D=0.475 \mathrm{Ns} / \mathrm{m}$.

2) Coulomb-damped resonant generator (CDRG). In this device, usually implemented with electrostatic forces on capacitor plates, the damping force is of constant magnitude, always opposing relative motion between the proof mass and the generator frame (i.e., $f=-F \cdot \operatorname{sgn}(\dot{z})$, where $F$ is constant) [18], [22].

An important characteristic of the mass-frame displacement waveform is that a Coulomb-force can cause the relative motion between the proof-mass and the frame to reduce to zero for a period of time [26] in which case the mass "sticks." During this phase, no energy is generated. This is shown for two stops per half-cycle, with the solid line in Fig. 2, where $m=1 \mathrm{~g}, k=80 \mathrm{~N} / \mathrm{m}$, and $F=0.95 \mathrm{mN}$. Sticking occurs when the mass has stopped relative to the frame and the absolute value of the Coulomb-force is larger than the absolute value of the sum of spring force and inertial force at that time. As soon as the mass starts to move, the Coulomb-force acts to oppose the motion and, during a sticking period, reverses the direction of the resulting relative acceleration. In simulation this causes the mass to oscillate around a stationary point relative to the generator frame. As the simulation time-step is decreased the amplitude
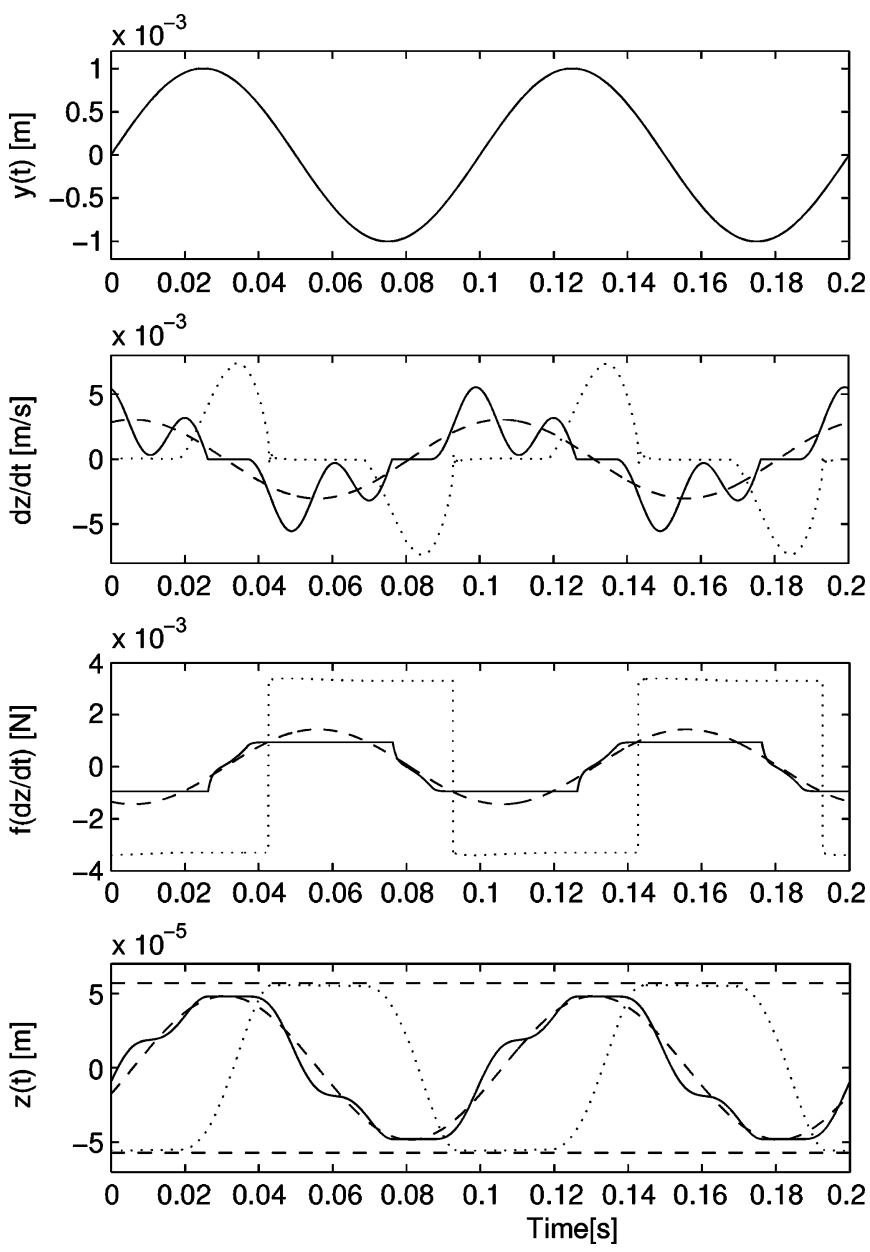

Fig. 2. Comparison of the operation of the three generator architectures. Dashed line is the VDRG, solid line the CDRG, and dotted line the CFPG. $\pm Z_{l}$ is shown as dashed straight lines.

and period of this oscillation approach zero. Fig. 2 shows the average force for the stationary periods in the cycle, as the results have been filtered to remove high frequency oscillation. In a practical CDRG, the capacitor being repeatedly charged and discharged would be lossy and consequently the device requires hysteresis around $\dot{z}(t)=0$ in order to reduce the frequency of charging. Thus, in a practical implementation, the mass would oscillate at reduced frequency but non-zero amplitude.

3) Coulomb-force parametric generator (CFPG). In this nonresonant device, again usually electrostatic in nature, the damping force (here also called holding force) is constant and holds the mass at one end of the travel (against an end-stop) until the input acceleration exceeds a threshold, thus ensuring that the distance between the limits is traveled under the highest possible force [25], [27]. The mass starts to move relative to the frame when the inertial force is great enough to overcome the holding force. Once the mass has reached the opposite side of the frame, the sign of the holding force is reversed and a new generation cycle starts. The operation of this generator is shown by the dotted line in Fig. 2 for $m=1 \mathrm{~g}$, $F=0.81 \mathrm{mN}$. 
Previous analysis of these three architectures using sinusoidal driving motions [25], i.e., $y(t)=Y_{0} \cos \omega t$, has shown that the CFPG achieves the highest power density of the three architectures when the amplitude of the driving motion $\left(Y_{0}\right)$ is much greater than the limit of the generator's internal travel $Z_{l}$. However, one of the main potential applications for microgenerators is the powering of medical electronics, where the driving motion for the generator is the motion of the human body. In this case, the driving motion is not sinusoidal and has a rich spectral content.

In this paper, we compare the optimal performance of these different architectures using actual measured acceleration waveforms from nine locations on the body of walking human subjects. The generated power is determined by calculating the power dissipated in the damper of the model of Fig. 1. Analytical solutions for generator performance with these measured acceleration signals are not feasible and, in addition, the spectral content across different human subjects differs making a general analysis difficult. Consequently, the approach presented here is numerical. Models for all three generator types have been implemented in Matlab. Operation of the resonant models was verified in Simulink [28] and the parametric generator (CFPG) model verified in the circuit simulator PSPICE. The generator parameters were then optimized.

The simulation models of the three architectures with measured acceleration data can be used to investigate several aspects of the optimization of microgenerators for realistic conditions. It is possible to compare which body locations yield the most power, which generator architecture is best suited to which body location and how this result depends on the size limitation of the generator. It is also possible to assess how sensitive the power yield is to variation in generator parameters from the optimal values or to variation in movement data from one subject to another.

It was shown in [25] that for sinusoidal driving motions, maximum power output from any architecture of generator is proportional to the value of the proof mass, assuming all other parameters are held constant. It can be shown that this proportionality holds for any shape of input waveform. Therefore, where appropriate in this paper, graphs of generated power have been normalized by the proof mass.

\section{DAta COLlection}

A custom-made wearable sensor system, PadNET [29], was used to acquire the acceleration signals. The system consists of three tri-axial sensor-modules based on ADXL210E (range $\pm 10 \mathrm{~g}$ ) and ADXL202E (range $\pm 2 \mathrm{~g}$ ) sensors [30], i.e., the system is able to record acceleration from all three axes from three independent locations at a time. Two ADXL sensors packaged in a small, light-weight PVC box form one sensor-module which has a substantially lower weight than typical commercial three-dimensional (3-D) acceleration sensors [31]. The acceleration signals were filtered by a second-order low pass filter $\left(f_{-3 \mathrm{~dB}}=55 \mathrm{~Hz}\right.$ ) before being sent to an iPAQ PDA which recorded the data with a sampling rate of $150 \mathrm{~Hz}$. The sensor modules were tightly fastened to the body using elastic straps
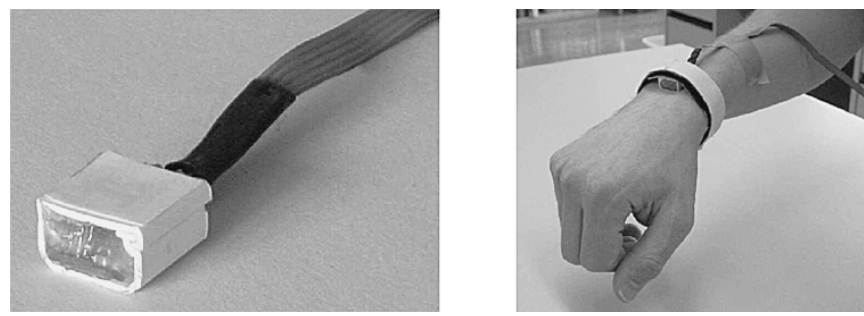

Fig. 3. Left: sensor module. Right: module mounted on wrist.

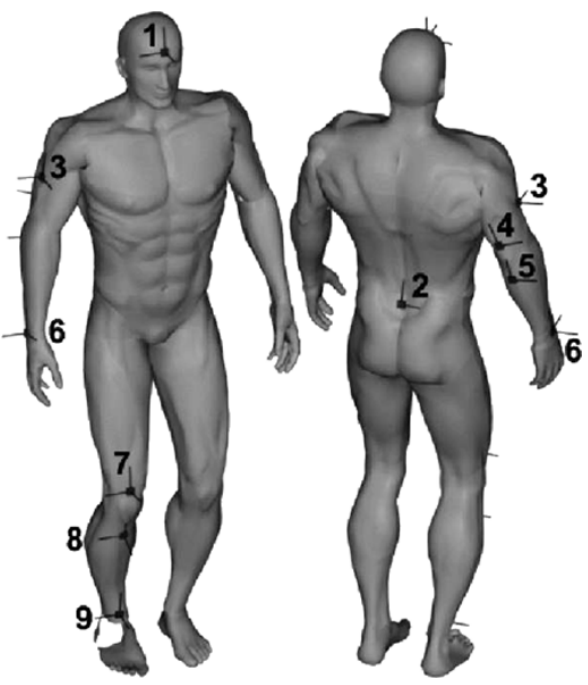

Fig. 4. Locations of measurement points on test subject (3-D model courtesy of Fred Dowling).

with velcro ends, as shown in Fig. 3, to ensure that there was little relative acceleration between the body and the sensor.

Eight test subjects [male, age: $27.3 \pm 2.5$ years, weight: 71.6 $\pm 7.4 \mathrm{~kg}$, height: $179 \pm 6.5 \mathrm{~cm}$ (mean \pm s.d.)] wearing normal walking shoes were asked to walk normally for $60 \mathrm{~s}$ on a treadmill running at constant $4 \mathrm{~km} / \mathrm{h}$, while the acceleration data was recorded. The sensor modules were attached to the nine locations shown in Fig. 4. Three independent measurements were taken for each location and each subject.

It is important to note that every recorded acceleration signal in this setup is based on a moving reference frame (i.e., moving acceleration sensor). Consequently, the orientation of the acceleration sensors cannot be determined without additional information from a gyro and so in this case it is not possible to compute the 3-D trajectory of the measurement points. However, by double integrating the recorded acceleration signals it is possible to obtain an equivalent position signal that, when fed into the generator model, leads to the same relative motion of the generator proof-mass as the actual body motion. In Fig. 5, an example acceleration waveform and its equivalent position waveform are shown.

\section{METHODOLOGY AND JUSTIFICATION}

\section{A. Processing of Acceleration Signals}

Orientation (i.e., in terms of azimuth and elevation) and, consequently, the oscillation axis of the proof-mass motion relative to the body can be selected to maximize energy yield. It was 

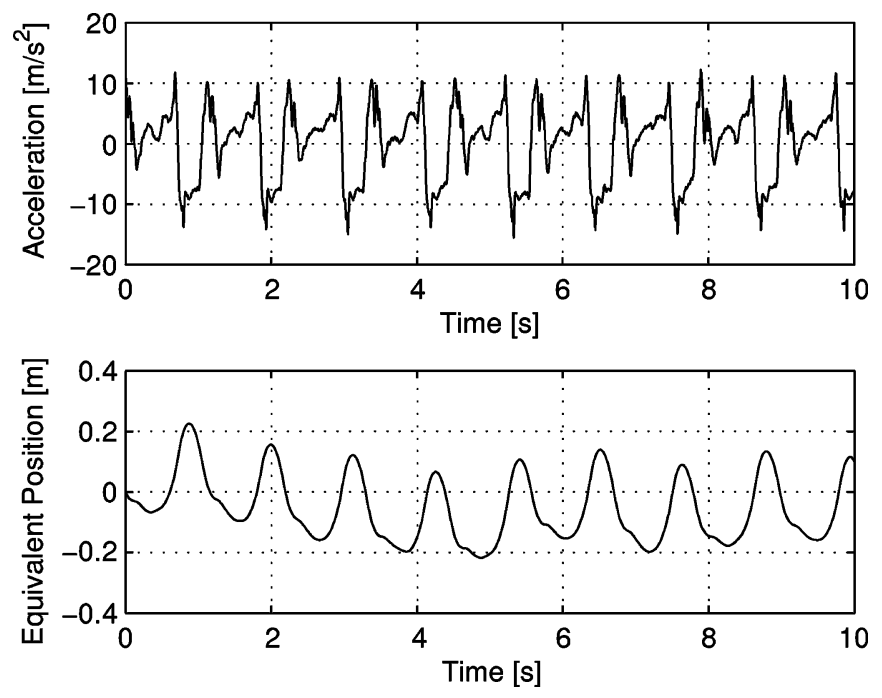

Fig. 5. Example acceleration waveform and equivalent position waveform (location 8).

decided to align the generator in a direction for which the acceleration signals had the highest variance, as this gives a reasonable indicator of the orientation which would provide maximum power. Thus, the 3-D acceleration signal from each location was reduced to a one-dimensional (1-D) signal in the chosen direction.

Simulations to determine the optimal parameters and maximum available power densities were performed on $10 \mathrm{~s}$ of data from one subject. The waveform data gathered from the other subjects was used to analyze the variations in generator performance across subjects as described in Section IV.

\section{B. Effects of Static Acceleration on Generator Performance}

The acceleration sensors measure both dynamic (ac) acceleration and static (dc) acceleration. Because the sensors are based on the measurement of the force acting on their proof-mass, gravity affects their output signal. Therefore, a dc component in the acceleration sensor signal can be due to the effect of gravity or a steady acceleration.

For the resonant generators, static acceleration affects the quiescent position of the mass within the frame, thus lowering the possible amplitude of relative mass-to-frame motion and consequently lowering the generated power. Ideally, static acceleration should be compensated out. This can be achieved by adding a constant offset force to the damping, i.e., using different damping forces in the up and down directions.

In this paper, it is assumed that the static acceleration can be compensated out by using different damping forces in each direction and so the 1-D acceleration signals were high-pass filtered with a third-order Butterworth filter with break frequency $0.4 \mathrm{~Hz}$.

\section{Optimization}

The resonant generator models have two parameters to be optimized: The resonant frequency (set by $k$ ) and the damping force ( $D$ for the VDRG and $F$ for the CDRG). The parameters were varied in the simulations to obtain maximum power while obeying the displacement constraint described in Section III-D. Because of the unknown shape of the optimization surface, and because an exhaustive full parameter search is too computationally intensive, adaptive simulated annealing (which is less likely than gradient methods to become stuck in a local minimum) has been used as the optimization method [32] for the resonant generators. As the CFPG has only one parameter to optimize (the value of the Coulomb-force $F$ ) the maximum power for the CFPG has been found by sweeping this parameter across a range.

The lower limits for the parameters of $k$ and $\omega_{n}$ were set to zero for the optimization. The upper limits chosen can be justified as follows. The CFPG and the CDRG generate energy until the Coulomb-force is increased to a point where the force is too large to allow any relative movement between the mass and frame. The value at which the Coulomb-force $F$ becomes large enough to stop relative mass-frame motion was the value used for the upper limits of the search space.

The upper limit of spring constant $k$ was set to a value which, even with very little damping, still limited the internal massframe displacement to less than $Z_{l}$. Care was taken to ensure that the resulting maximum undamped resonant frequency $\left(\omega_{n}=\right.$ $\sqrt{\mathrm{k} / \mathrm{m}}$ ) was well above the bandwidth of the input motion. This guarantees that the optimal value of $k$ is included in the search space.

The same argument was used to set an upper limit on the value of velocity damping coefficient $D$. The damping coefficient should not be greater than that which restricts the motion of the mass from moving the distance $2 Z_{l}$ in one period, because in an optimal configuration, the mass will likely make full use of the allowed travel.

\section{End-Stop Limited Travel}

The collision of the proof mass with the generator frame has different effects upon the different architectures.

- CFPG: This device is designed to operate with the proofmass snapping backward and forward between end-stops. If the holding force (Coulomb force) has been set optimally for a particular flight, the mass arrives at the opposite side of the generator frame with zero velocity. If the holding force is set below the optimal value then the mass simply arrives at the opposite side of the frame with a non-zero velocity, and some kinetic energy will be lost in the impact.

Because the modeling of this collision is very dependent upon the generator type, structure and materials used, the simple assumption used in this work is that the relative mass-frame velocity becomes zero upon impact. This may lead to an underestimate of the generated energy.

- VDRG and CDRG: While a resonant generator may indeed work if the proof-mass collides with the constraining end-stops, it is no longer acting in a resonant mode. Thus, solution sets for which the resonant generators exceed the displacement constraint are ignored. 
TABLE I

GENERATOR CONFIGURATIONS USED

\begin{tabular}{c|c|c}
\hline Config N $^{o} \cdot$ & $\mathrm{m}[\mathrm{g}]$ & $\mathrm{Z}_{l}[\mathrm{~mm}]$ \\
\hline 1 & 0.1 & 0.05 \\
\hline 2 & 0.3 & 0.25 \\
\hline 3 & 0.5 & 1 \\
\hline 4 & 1 & 5 \\
\hline 5 & 2 & 20 \\
\hline
\end{tabular}

\section{E. Generator Configurations}

The generator configurations in Table I were used in the simulations for all generator architectures, in order to investigate the effect of size on generator performance.

Configurations 1 and 2 correspond to MEMS implementations of microgenerators, with 1 being smaller than reported configurations, and 2 being the typical size of previously reported generators. Configurations 3-5 correspond to generators which could be fabricated using standard precision engineering techniques. Configuration 5 is larger than could be tolerated in many suggested applications, but is included for comparison purposes and to give a broader indication of generator scaling effects.

\section{F. Measurement Noise in Acceleration Signals}

In order to ensure that noise in the acceleration signals was not a large contributing factor to the power generated, data from stationary sensors was recorded and the same optimization process was carried out as previously for the actual measured waveforms. In most cases, the power generated from these signals is negligible compared to the power generated from the signals from the walking motion. The exception is the smallest generator configuration used (configuration 1), where, in the worst case, the power generated from the noise rises to $35 \%$ of the power obtained from walking motion. However, the median for configuration 1 is only $5 \%$.

\section{PERformance COMPARISON}

The performance metric for microgenerators is described by the product of two terms [25]: the coupling effectiveness (coupled power/maximum possible coupled power) and an efficiency (useful power output/coupled power). Coupled power is the amount of power dissipated in the damper, i.e., coupling effectiveness gives an indication of how much power the damper extracts from the source (which in turn is the upper limit for the generated electrical power). Useful power output is the power available to drive external circuitry after suitable power processing, and is beyond the scope of this paper.

The plots of Fig. 6 show the maximum coupled power from 2 different sizes of generator, after optimization (i.e., when the coupling effectiveness is equal to 1 ). Results are given for all the three architectures on each of the nine measured locations on the body. Fig. 6(a) shows the maximum coupled power for configuration 2, and Fig. 6(b) shows the performance of the larger configuration 5. Locations 2-6 are on the upper body, and 7-9 the lower body, with location 1 being on the head.

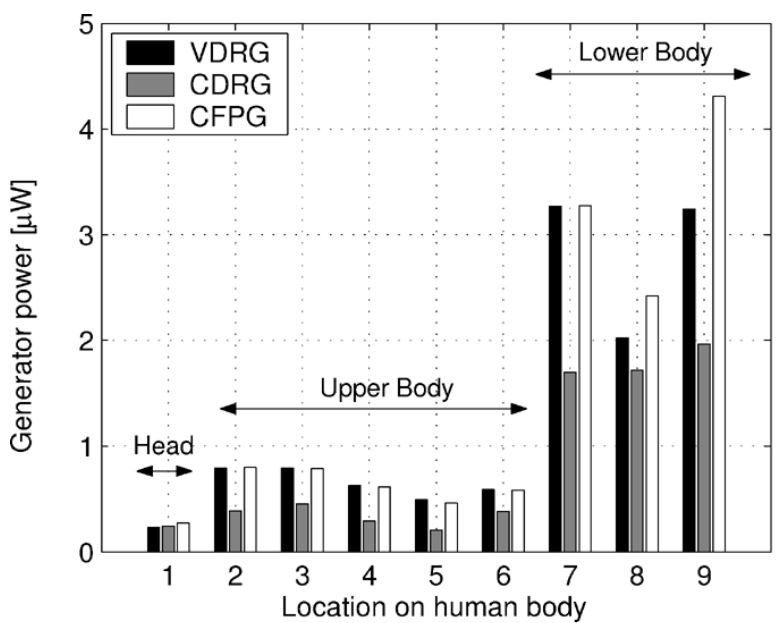

(a)

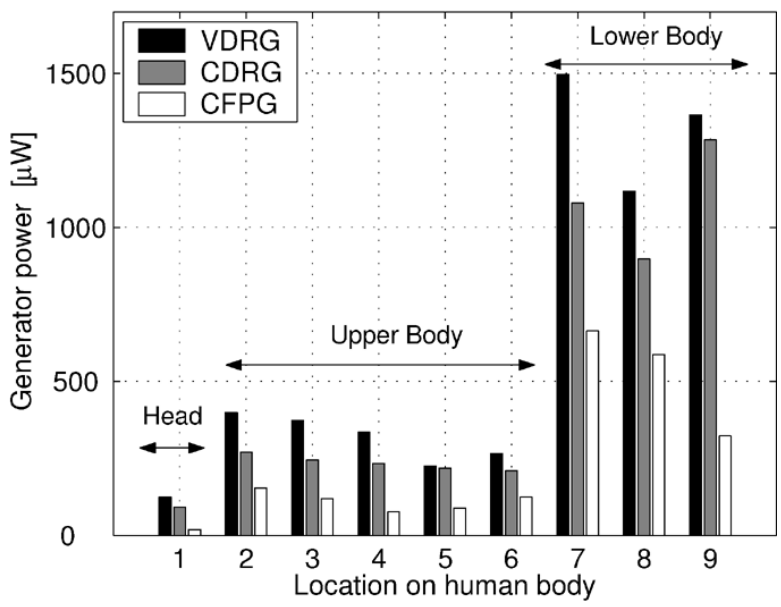

(b)

Fig. 6. Generator architecture absolute performance. (a) Configuration 2 $\left(Z_{l}=250 \mu \mathrm{m}, \mathrm{m}=300 \mathrm{mg}\right)$. (b) Configuration $5\left(Z_{l}=20 \mathrm{~mm}, \mathrm{~m}=2 \mathrm{~g}\right)$.

For inertial generators under walking conditions, the lower body locations yield around four times more power than the upper body locations. This is to be expected because the accelerations on the lower body have higher RMS values.

Fig. 6(a) and (b) shows that optimized generator performance on locations 2-6 (on the upper body and the sacrum) are similar, and performance from locations 7-9 (on the knee and lower leg) are also similar. Consequently, in order to reduce the complexity of some of the following plots, two data sets have been formed, with the power equal to the mean of the values of the set from the various locations. The sets are: "upper body," consisting of locations 2-6 and "lower body" consisting of locations 7-9.

As can be seen, the CFPG is a marginally better choice for small generator sizes for most locations. As the generators increase in size, the resonant devices start to outperform the CFPG. The CDRG is never as good as the VDRG. The VDRG is the best choice for the larger scale devices.

In Fig. 7, the averaged normalized power values have been plotted against $Z_{l}$ for the two data sets. By interpolating between the computed points it is possible to estimate the generator dimension (represented by $Z_{l}$ ) where the resonant devices start to outperform the CFPG. For the upper body, this cross-over 


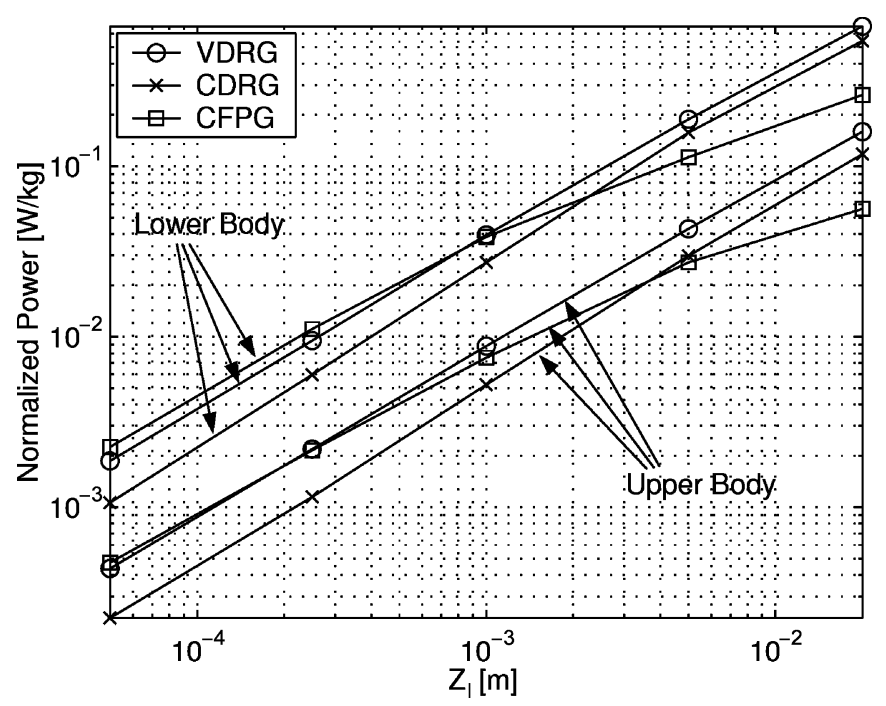

Fig. 7. Comparison of architecture performance for generators mounted on the upper and lower body (power normalized by the proof mass, $m$ ).

point is located at about $200 \mu \mathrm{m}$ and for the lower body at about $800 \mu \mathrm{m}$.

\section{OPTIMAL PARAMETERS}

The variation in the optimal generator damping parameters with generator size is shown in Fig. 8. As with the plots for generator output power, the VDRG damping coefficient $D$, and the Coulomb-force, $F$, have been normalized to the value of the proof mass (because the optimal trajectory is maintained for constant $D / m$ and $F / m$ values). It should be noted that the relationships between optimal damping, optimal spring constant and $Z_{l}$ discussed in this section are solely based upon the five generator sizes which have been simulated.

Fig. 8(a) shows the variation of the damping coefficient $D$ with $Z_{l}$ for the VDRG. Over much of the range, the data forms a straight line on a log-log plot, and over that range, $D \propto 1 / Z_{l}$. Once $Z_{l}$ exceeds $5 \mathrm{~mm}$, this relation no longer holds for all positions.

Fig. 8(b) shows the normalized Coulomb-Force for the CDRG. As can be seen, for a given location, the force is approximately constant for $Z_{l}<5 \mathrm{~mm}$ before starting to decrease for some locations.

Fig. 8(c) shows the dependence of the optimal holding force of the CFPG on $Z_{l}$. It should be noted that as the value of $Z_{l}$ increases, it is expected that the optimal holding force would reduce to allow the mass to travel further in one flight.

Fig. 9 shows the dependence of the optimal resonant frequencies on generator size. Fig. 9(a) shows the variation of optimal resonant frequency for the VDRG. For $Z_{l}<1 \mathrm{~mm}$, $\omega_{n} \propto 1 / \sqrt{Z_{l}}$, i.e., $k \propto 1 / Z_{l}$. When $Z_{l}$ is larger than this, the relation no longer holds for all locations, with resonant frequencies rising above that predicted by this relationship.

Fig. 9(b) shows the dependence of the CDRG optimal resonant frequency upon the value of $Z_{l}$. While these lines are not completely parallel, the relationship between the $Z_{l}$ and the optimal resonant frequency is approximately $k \propto 1 / Z_{l}$, i.e., the same as the VDRG.

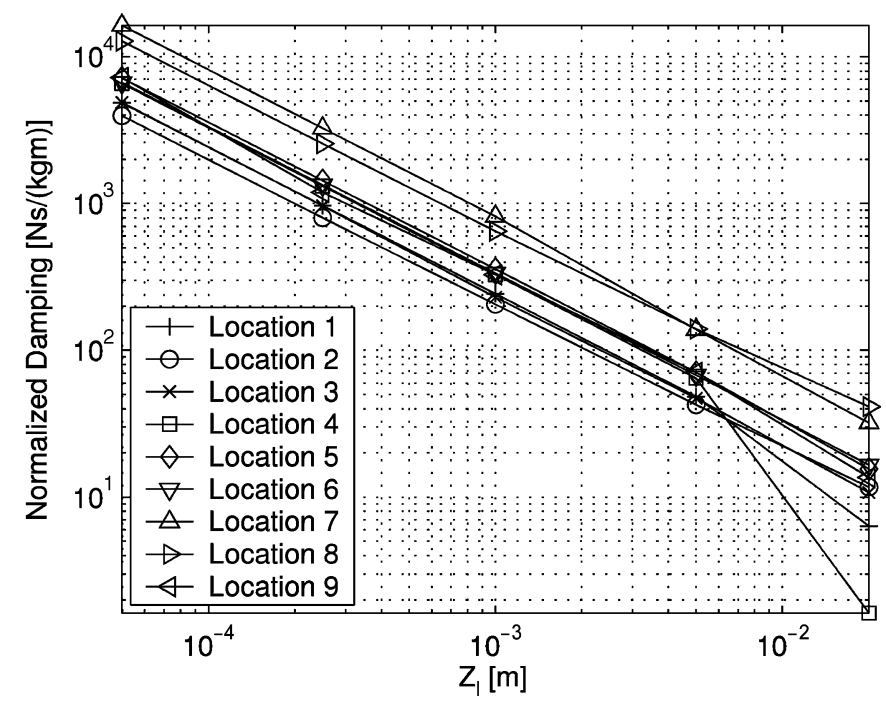

(a)

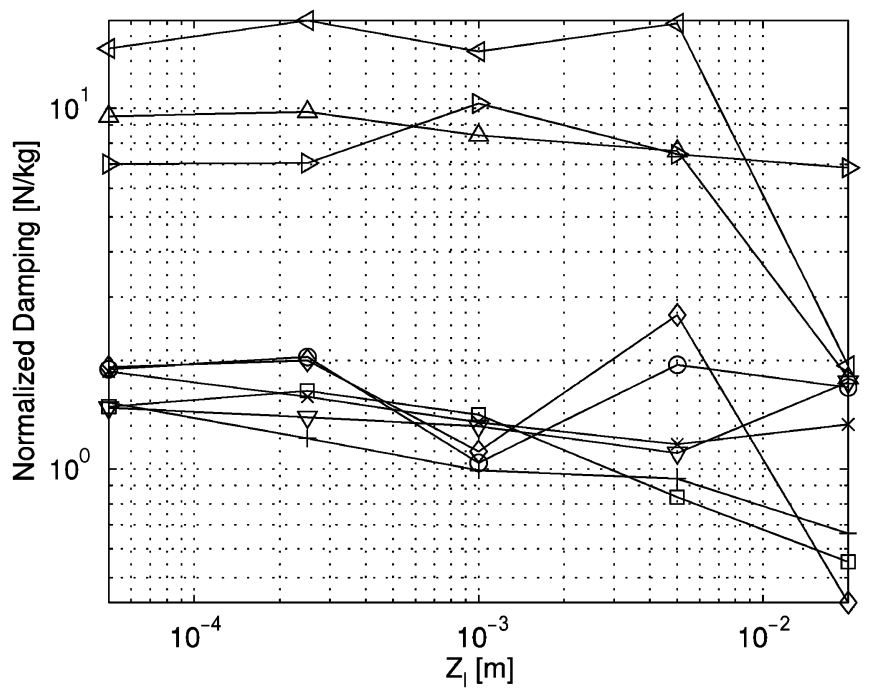

(b)

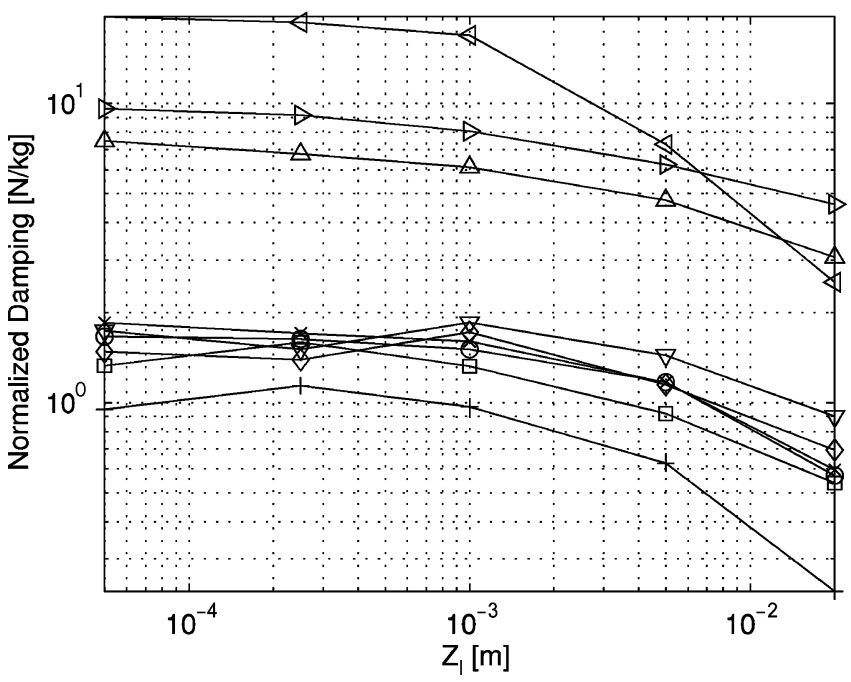

(c)

Fig. 8. Optimal generator damping. (a) Optimal VDRG damping coefficient. (b) Optimal CDRG damping force. (c) Optimal CFPG damping force. 


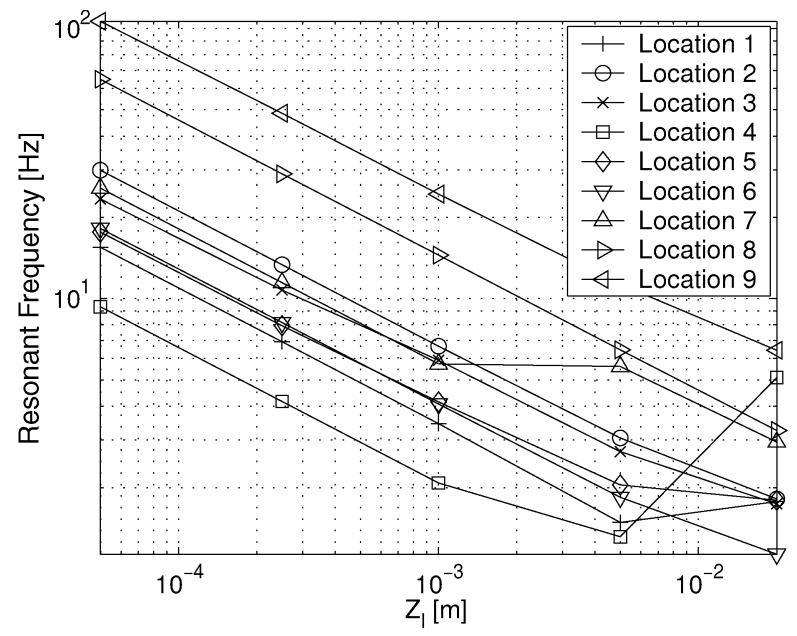

(a)

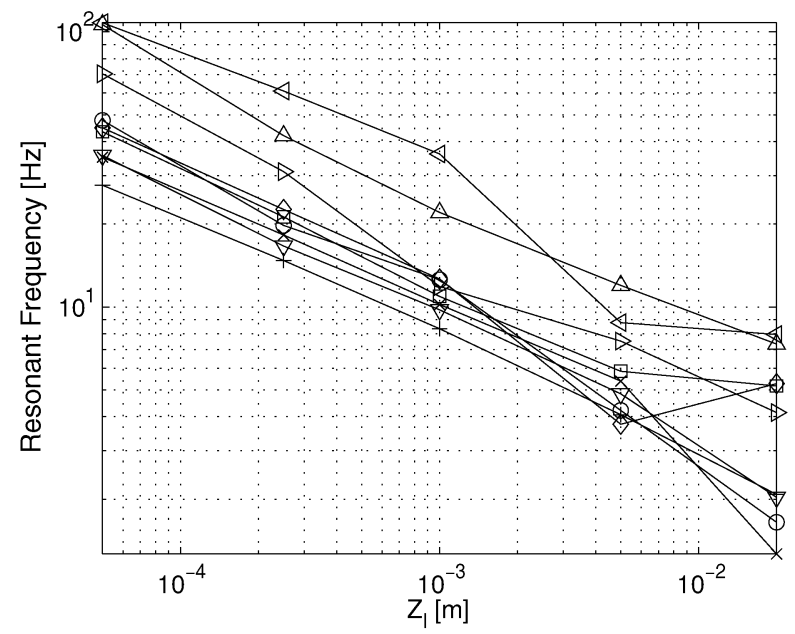

(b)

Fig. 9. Optimal generator resonant frequency. (a) Optimal VDRG resonant frequency. (b) Optimal CDRG resonant frequency.

The changes in the relationships between the parameters (i.e., a proportional relationship until $Z_{l}$ exceeds a certain value followed by a different choice of optimal parameter) suggests that the optimal operation for the generators shifts between modes, which correspond to the system changing from being overdamped or underdamped, i.e., once $Z_{l}$ has exceeded $10^{-3} \mathrm{~m}$, resonant amplification is possible. This is shown in Fig. 10, with the resonant generators producing an output which is much closer to a sinusoid for configuration 5 (which operates underdamped) than configuration 2 (which operates overdamped).

\section{Sensitivity of Power to Generator Parameters AND INTER-SUBJECT VARIATIONS}

It is important to consider the sensitivity of power output to changes in generator parameters. First, the measured data is for a narrow range of walking speed, and the resonant frequency will probably not be dynamically adjustable. By varying the spring constant in the simulation we can alter the relation between resonant frequency and walking speed, which, assuming a similar harmonic content, gives a reasonable approximation to
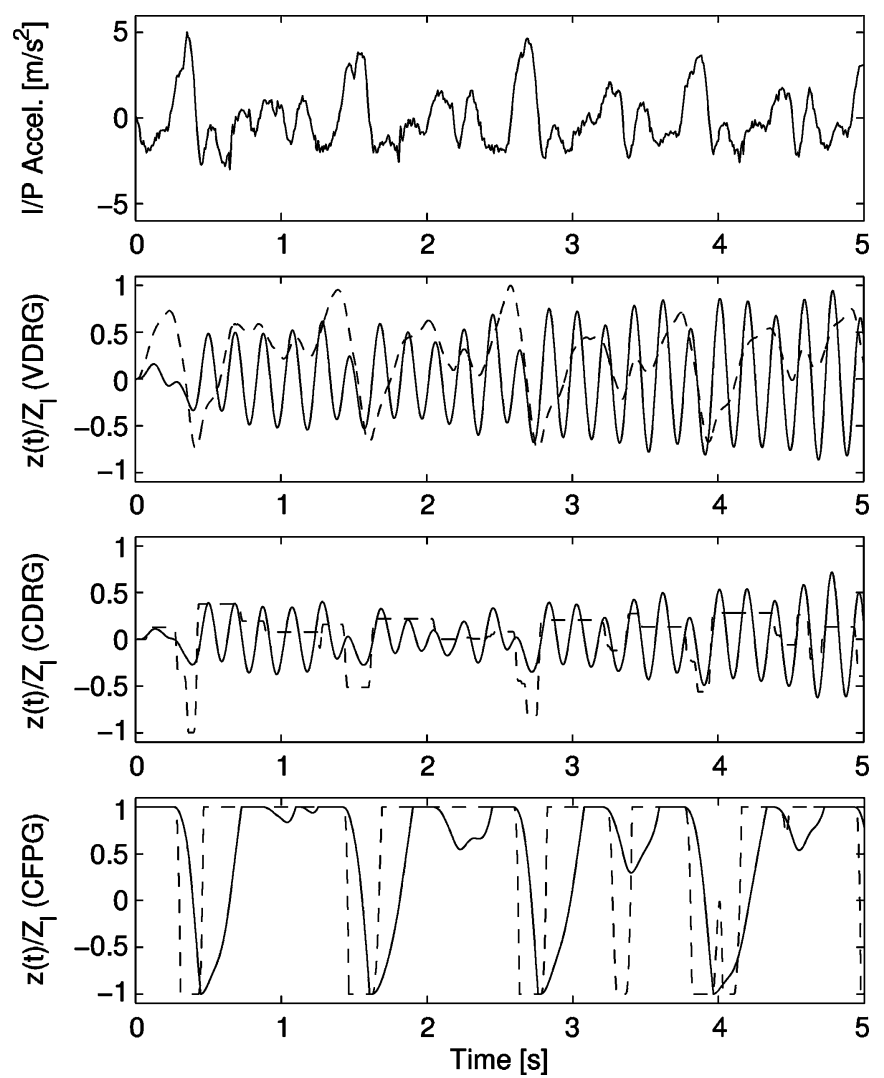

Fig. 10. Relative position waveforms for two sizes of each architecture for one input acceleration waveform. The dashed line is configuration 2. and the solid line configuration 5 .

varying walking speed. Although the damping coefficient may be dynamically varied, how precisely it is optimized will depend on the control circuitry and other factors so the dependence of power on the deviation of damping from optimality is also of interest.

Therefore, the parameters of spring constant (for resonant generator types only) and damping term (for all generator types) were varied. For every optimum, both parameters were varied in seven discrete steps to deviate $\pm 20 \%$ from the optimal value. The results have been grouped and averaged for the upper body and the lower body groups as before.

The damping parameter is regarded as adjustable online during generator operation and consequently when varying the spring constant, the damping parameter was re-optimized each time. In contrast, the spring constant is regarded as fixed during generator operation and was, hence, held constant during the variation of the damping parameter.

In Fig. 11, the generator coupling effectiveness for two generator sizes (configuration 2, small, and configuration 5, large) is shown. For the resonant generator types, points with damping parameter values below the optimum are not shown because they all violate the displacement constraint.

For most cases, generated power does not fall more than $20 \%$ for a deviation of $20 \%$ in parameters from optimal. Exceptions are the CDRG for the upper body and the CFPG with an above optimal damping parameter. For the CFPG, this is due to the fact that the number of flights of the proof-mass is strongly reduced with the damping force above optimal, while below the 


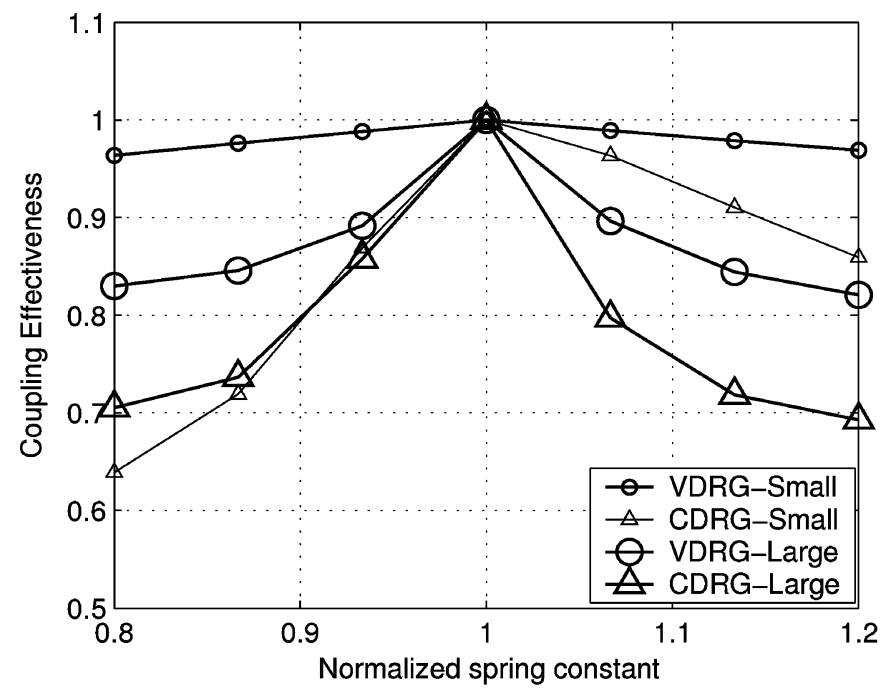

(a)

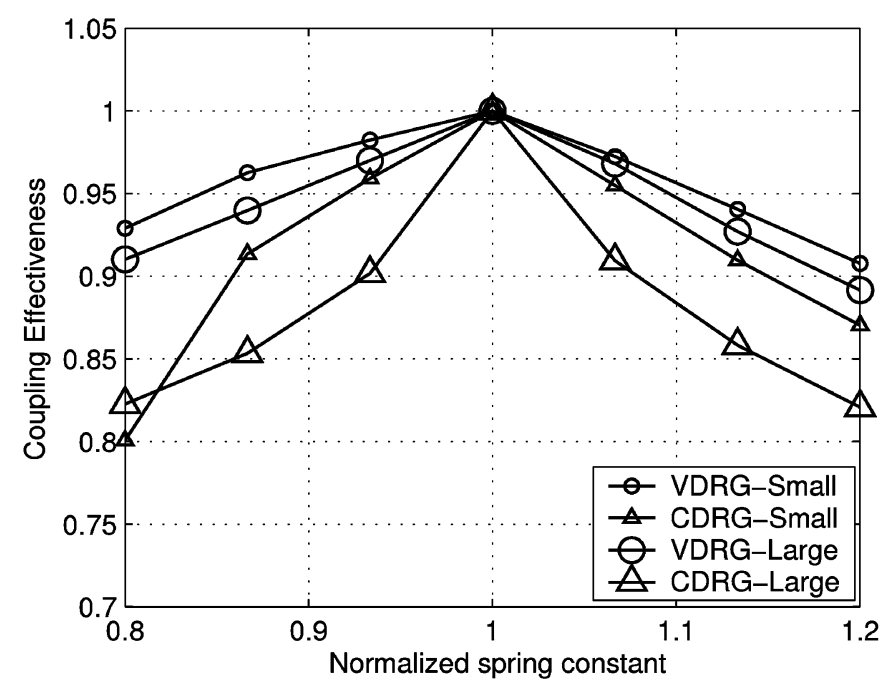

(c)

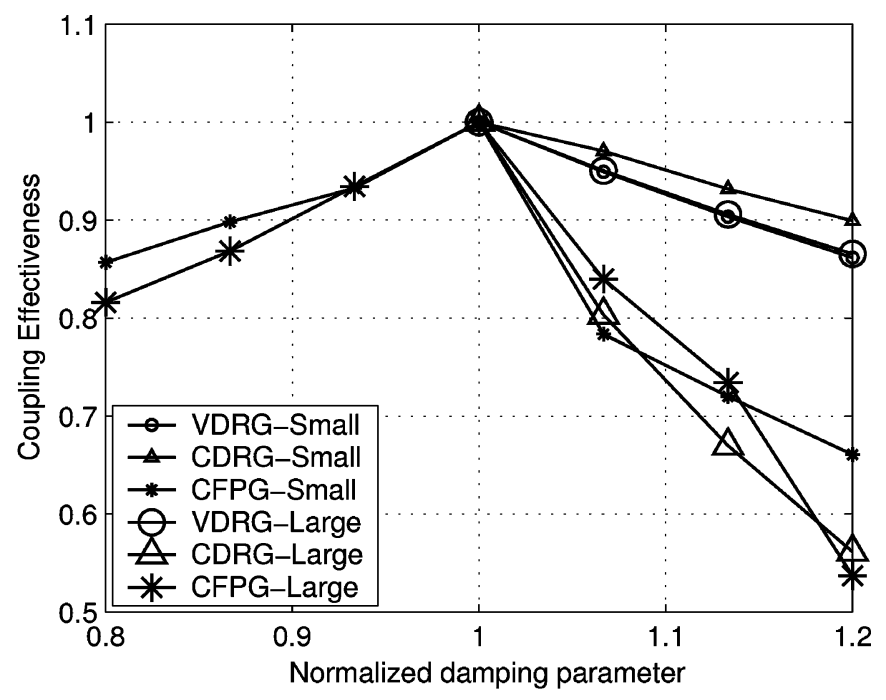

(b)

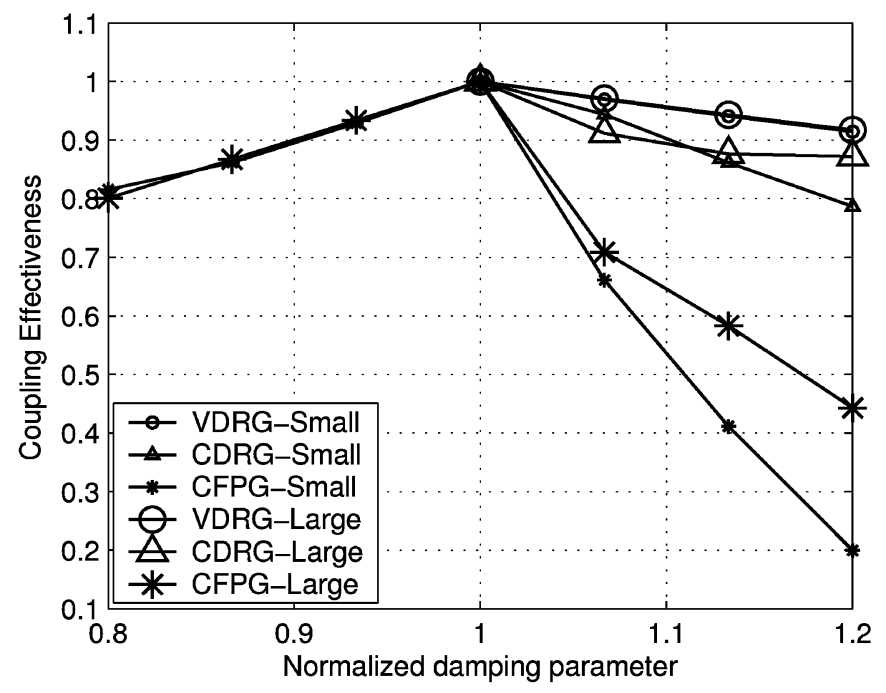

(d)

Fig. 11. Sensitivity of coupling effectiveness to changes in generator parameters. (a) Coupling effectiveness with deviation in k, upper body. (b) Coupling effectiveness with deviation in damping, upper body. (c) Coupling effectiveness with deviation in k, lower body. (d) Coupling effectiveness with deviation in damping, lower body.

optimum the generated power is approximately proportional to the damping force. For the CDRG, the reason may be due to an increase in the number of times the mass motion sticks relative to the frame.

As mentioned in Section III-A, the generators have been optimized for the input waveforms of one subject. However, the variations in generator performance across subjects have also been considered. This was done by feeding acceleration waveforms from the seven other subjects into the optimized generator models. The damping parameter was re-optimized for every waveform since it is considered adjustable online during generator operation. The output power values for the other subjects were comparable to the ones of the main subject (same order of magnitude, typically not deviating more than $\pm 50 \%)$.

While the CFPG has the inherent advantage of not having a resonant frequency and, therefore, offering the potential of being fully optimized dynamically to suit the operating condi- tions, Fig. 11(a) and (b) shows that the loss of power for the small VDRG, for example, with loss of tuning is not rapid.

\section{PRACTICAL CONSIDERATIONS}

An important question is what minimum generator size and mass would be needed to realize the required parameters found in Section V. Here, the issues are only briefly discussed, as an in-depth analysis is beyond the scope of this paper. A simple realization of a VDRG is a cylindrical coil moving in and out of a homogeneous magnetic field region and connected to a resistive load. The Lorentz force that occurs is the damping force. A CDRG/CFPG can be implemented by a parallel plate capacitor, where one plate is movable. Here, the damping force is the electrostatic force between the capacitor plates. There are two basic types: The gap-opening type is operated in constant charge mode and the sliding-plates type is operated in constant-voltage mode. For these configurations, it can be shown that when all 
dimensions are scaled by the same factor the VDRG force is proportional to $L^{3}$ while the CDRG/CFPG force is proportional to $L^{2}$, where $L$ is the dimension of length. Hence, for decreasing sizes, the electromagnetic damping forces drop more rapidly than the electrostatic ones and it becomes increasingly difficult for the former to achieve the required damping to operate optimally.

In order to adapt to different driving motions, the damping force needs to be adjustable online. In an electromagnetic VDRG implementation this can be done by changing the load resistance. In electrostatic generators the damping force is controlled via the voltage of the capacitor.

As stated in Section III-B, it was assumed that static acceleration can be compensated out by adding a constant offset force. While this is difficult to do for the electromagnetic and piezoelectric generators, it is much easier to achieve for electrostatic generators, i.e., the CDRG and the CFPG. In electrostatic generators, the offset force can easily be realized by using different electrostatic forces in the up and down directions, which in turn can be achieved by pre-charging the capacitor to different values depending upon the relative direction of travel of the mass.

Another practical issue is startup. While an electromagnetic generator based on permanent magnets can start up with zero electrical energy stored, an electrostatic generator needs a minimum energy to charge the capacitor. In principle, it is possible to use an electret to precharge the capacitor [33]; however, in that case, the damping force is not easily adjustable.

\section{DISCUSSION}

Fig. 7 shows that for human walking movements, the generator architecture with the highest power density is dependent upon generator size and the position on the body on which the generator is mounted. The CFPG is the optimal architecture on the lower body when $Z_{l}<800 \mu \mathrm{m}$ and the VDRG for $Z_{l}>800 \mu \mathrm{m}$. For the upper body, the architecture choices are the same, but the cross over point is at $200 \mu \mathrm{m}$.

For sinusoidal motion, the cross-over point between architectures can be defined in terms of the ratio of $Z_{l} / Y_{0}$ and $\omega / \omega_{n}$ [25], where $Y_{0}$ is the amplitude of the driving motion. With inputs containing multiple frequencies, this distinction cannot easily be made because $Y_{0}$ and $\omega$ have no clear meaning. However, it can be noted that the displacements on the upper body are smaller than the lower body. So, it is reasonable to expect that the value of $Z_{l}$ for which there is a change in optimal architecture is smaller for the upper body locations.

The achievable power density for inertial microgenerators does not remain constant for changes in generator size (specifically for changes in $Z_{l}$ ) [25] and so absolute power densities cannot be quoted. For optimal implementations of resonant microgenerators with sinusoidal inputs, the volume of the proof mass will be half of the generator volume [25] (because the generated power is proportional to both $m$ and $Z_{l}$ ). While this does not hold exactly for nonsinusoidal motion, Fig. 7 shows that for all architectures and for $Z_{l}<1 \mathrm{~mm}$, the power is approximately proportional to $Z_{l}$. Consequently, setting the mass to occupy half the volume is a good approximation to obtaining the highest power density for small generators. However, this assumption will underestimate the maximum power density for the larger generators.

The achievable power densities have been calculated for the sizes of generator studied in this paper based upon the assumption that the mass occupies half the volume. With $Z_{l}= \pm 250 \mu \mathrm{m}$ this gives a total length of the generator (in the direction of mass motion) of $1 \mathrm{~mm}$, allowing for the height of the mass. Assuming the generator is cube shaped, then the total volume of an optimal mass, made of gold, is $0.5 \mathrm{~mm}^{3}$, giving a total mass of $10 \mathrm{mg}$. This gives power densities of $140 \mu \mathrm{W} / \mathrm{cm}^{3}$ for location 9 , and $8.7 \mu \mathrm{W} / \mathrm{cm}^{3}$ for location 1, for CFPGs. For $Z_{l}= \pm 5 \mathrm{~mm}$, the power density is $2100 \mu \mathrm{W} / \mathrm{cm}^{3}$ for location 9 and $134 \mu \mathrm{W} / \mathrm{cm}^{3}$ for location 1, in this case, for VDRGs.

For wearable applications, sensor nodes will usually be monitoring environmental conditions or biological functions and, thus, have modest data rate requirements. For example, heart rate monitoring has been estimated to require only $80 \mathrm{bits} / \mathrm{min}$ [34]. It can be shown that transmission at up to $1 \mathrm{kbit} / \mathrm{s}$ over a 1-m range can be provided with submicrowatt power levels [35]. Recently, A-D converters have been reported with power consumption of $1 \mu \mathrm{W}$ with sampling rates above the requirements for health monitoring applications. This suggests that power levels as low as $1 \mu \mathrm{W}$ may be sufficient for realistic sensor nodes. Consequently the minimum size of a microgenerator for use on the human body to allow it to drive a sensor and wireless link lies between $1 \mathrm{~mm}^{3}$ and $1 \mathrm{~cm}^{3}$ depending on the location of the generator on the body, and the duty cycles of the motion and the sensor operation.

\section{CONCLUSION}

The simulated performance of the three microgenerator architectures has been investigated with the input motion being human walking motion. Acceleration data were measured from nine body locations on eight human test subjects. One test subject was chosen and the generator parameters were optimized on $10 \mathrm{~s}$ of the data from that subject for all body locations. Five different sizes of generator have been investigated and their optimal parameters have been plotted against generator size. For the resonant generators with $Z_{l}$ values less than $1 \mathrm{~mm}$, the optimal values of spring constant and damping are proportional to $1 / Z_{l}$. For $Z_{l}>1 \mathrm{~mm}$, the optimal values no longer obey this proportionality, which suggests a change in the mode of generator operation.

The generated power for generators where $Z_{l}<1 \mathrm{~mm}$ is proportional to $Z_{l}$. For generators with larger $Z_{l}$, this relationship breaks down, with generated power increasing less rapidly with $Z_{l}$. This suggests that as generators get large, an optimal microgenerator configuration will contain a proof-mass which occupies more than half the volume of the generator. The maximum power output of a generator for a given input and value of $Z_{l}$ is proportional to the value of the proof mass and, thus, optimal values of the parameters, $D, F$, and $k$ also scale with the value of proof mass. 
It has been shown that the CFPG is the architecture which achieves the highest power density for smaller generators and the VDRG for larger generators, with the cross over points being $Z_{l}=200 \mu \mathrm{m}$ for generators mounted on the upper body and $Z_{l}=800 \mu \mathrm{m}$ for the lower body. The CDRG did not outperform the other two architectures for any location or for any size of generator. The scaling laws of electrostatic forces and electromagnetic forces also demonstrate the suitability of the CFPG to small generators and the VDRG to larger generators. There is a distinct difference between the energy generated from upper and lower body locations for walking motion, with there being approximately four times as much energy available from the lower body locations. However, it should be noted that many sensors, especially implanted medical sensors, would need to be mounted on specific locations, and, hence, it might not be possible to place them at the locations which achieve the highest power densities.

The coupling effectiveness of the VDRG is less sensitive to changes in spring constant than is the coupling effectiveness of the CDRG. The coupling effectiveness of the CFPG is less sensitive to a reduction of the damping force than to an increase. When, for a given optimal spring constant, the damping parameter for the resonant generators is set to below the optimum, the proof-mass hits the end stops, which shows that under optimal operation, the resonant generators operate to the displacement limit.

\section{ACKNOWLEDGMENT}

The authors would like to thank Dr. P. Miao at Imperial College London, for his helpful contributions on generator realization, and Dr. B. H. Stark of Imperial College London and Dr. P. Lukowicz of ETH Zurich for helpful discussions.

\section{REFERENCES}

[1] D. Wanless, "Securing our future health: Taking a long term view," HM Treasury, U.K., Apr. 2002.

[2] A. Flowerday and R. Smith, "Lessons learnt from long-term chronic condition monitoring," presented at the Int. Workshop on Wearable and Implantable Body Sensor Networks, London, U.K., Apr. 2004.

[3] P. Needham and L. Gamlyn, "Arrhythmia analysis in the community," presented at the Int. Workshop on Wearable and Implantable Body Sensor Networks, London, U.K., Apr. 2004.

[4] L. Gamlyn, P. Needham, S. M. Sopher, and T. J. Harris, "The development of a neural network-based ambulatory ECG monitor," Neur. Comput. Appl., vol. 41, no. 4, pp. 273-278, Apr. 1999.

[5] P. Bauer, M. Sichitiu, R. Istepanian, and K. Premaratne, "The mobile patient: wireless distributed sensor networks for patient monitoring and care," in Proc. IEEE EMBS Int. Conf. Information Technology Applications in Biomedicine, Arlington, VA, Nov. 2000, pp. 17-21.

[6] G. D. Abowd, "Ubiquitous computing: Research themes and open issues from an applications perspective," Georgia Inst. Technol., Atlanta, Oct. 1996.

[7] M. Weiser, "The computer for the twenty-first century," Sci. Amer, pp. 94-104, Sep. 1991.

[8] P. Lukowicz, J. A. Ward, H. Junker, M. Stäger, G. Tröster, A. Atrash, and T. Starner, "Recognizing workshop activity using body worn microphones and accelerometers," in Proc. 2nd Int. Conf. Pervasive Computing, Vienna, Austria, Apr. 2004, pp. 18-32.
[9] L. Bao and S. S. Intille, "Activity recognition from user-annotated acceleration data," in Proc. 2nd Int. Conf. Pervasive Computing, Vienna, Austria, Apr. 2004, pp. 1-17.

[10] M. Strasser, R. Aigner, M. Franosch, and G. Wachutka, "Miniaturised thermoelectric generators based on poly-Si and poly-SiGe surface micromachining," in Proc. 11th Int. Conf. Solid-State Sensors and Actuators, Munich, Germany, Jun. 2001, pp. 535-542.

[11] J. J. Kieley, D. V. Morgan, D. M. Rowe, and J. M. Humphrey, "Low cost miniature thermoelectric generator," Electron. Lett., vol. 27, no. 25, pp. 2332-2334, Dec. 1991.

[12] J. B. Lee, Z. Chen, M. G. Allen, A. Rohatgi, and R. Arya, "A high voltage solar cell array as an electrostatic MEMS power supply," in Proc. IEEE Workshop Microelectromechanical Systems, 1994, pp. 331-336.

[13] J. F. Randall, "On the Use of Photovoltaic ambient energy sources for powering indoor electronic devices," Ph.D. dissertation, School Eng. (STI), Swiss Fed. Inst. Technol., Lausanne, Switzerland, Jul. 2003.

[14] S. Suzuki, T. Katane, H. Saotome, and O. Saito, "A proposal of electric power generating system for implanted medical devices," IEEE Trans. Magn., vol. 35, no. 5, pp. 3586-3588, Sep. 1999.

[15] J. Kymissis, C. Kendall, J. Paradiso, and N. Gershenfeld, "Parasitic power harvesting in shoes," in Proc. 2nd Int. Symp. Wearable Computing, 1998, pp. 132-139.

[16] R. Amirtharajah and A. P. Chandrakasan, "Self-powered low power signal processing," in Proc. Symp. VLSI Circuits, 1997, pp. 25-26.

[17] R. Tashiro, N. Kabei, K. Katayama, Y. Ishizuka, F. Tsuboi, and K. Tsuchiya, "Development of an electrostatic generator that harnesses the motion of a living body," JSME Int. J., ser. C, vol. 43, no. 4, pp. 916-922, 2000.

[18] S. Roundy, P. K. Wright, and K. S. Pister, "Microelectrostatic vibration-to-electricity converters," in Proc. ASME Int. Mechanical Engineering Congress and Exposition, New Orleans, LA, Nov. 2002.

[19] N. M. White, P. Glynne-Jones, and S. Beeby, "A novel thick-film piezoelectric micro-generator," Smart Mater. Struct., vol. 10, pp. 850-852, Aug. 2001.

[20] J. Y. Kang, H. J. Kim, J. S. Kim, and T. S. Kim, "Optimal design of piezoelectric cantilever for a micro power generator with microbubble," in Proc. 2nd Annu. Int. IEEE-EMBS Special Topic Conf. Microtechnologies in Medicine and Biology, May 2002, pp. 424-427.

[21] M. J. Konak, I. G. Powlesland, S. P. van der Velden, and S. C. Galea, "A self-powered discrete time piezo-elecric vibration damper," presented at the Proc. SPIE, vol. 3241, 1997, pp. 270-279.

[22] S. Meninger, J. O. Mur-Miranda, R. Amirtharajah, A. P. Chandrakasan, and J. H. Lang, "Vibration-to-electric energy conversion," IEEE Trans. Very Large Scale Integr. (VLSI) Syst., vol. 9, no. 1, pp. 64-76, Feb. 2001

[23] S. Roundy, P. K. Wright, and J. M. Rabaey, Energy Scavenging for Wireless Sensor Networks. Boston, MA: Kluwer, 2003.

[24] T. Starner and J. A. Paradiso, "Human generated power for mobile electronics," in Low Power Electronics Design, C. Piquet, Ed. Boca Raton, FL: CRC, 2004.

[25] P. D. Mitcheson, T. C. Green, E. M. Yeatman, and A. S. Holmes, "Architectures for vibration-driven micropower generators," IEEE/ASME J. Microelectromech. Syst., vol. 13, no. 3, pp. 429-440, June 2004.

[26] J. P. Den Hartog, "Forced vibrations with combined coulomb and viscous friction," Trans. ASME J. Appl. Mech., vol. 53, pp. 107-115, Dec. 1931.

[27] P. D. Mitcheson, P. Miao, B. H. Stark, E. M. Yeatman, A. S. Holmes, and T. C. Green, "MEMS electrostatic micro-power generator for low frequency operation," Sens. Actuators A, vol. 115, pp. 523-529, 2004, to be published.

[28] (2002, Jul.) Matlab. [Online]. Available: http://www.mathworks.com

[29] H. Junker, P. Lukowicz, and G. Tröster, "PadNET: wearable physical activity detection network," in Proc. 7th Int. Symp. Wearable Computers, Oct. 2003, pp. 244-245.

[30] Analog Devices. (2000) ADXL202/210 iMEMS Accelerometer Datasheet, Rev. A. [Online]. Available: http://www.analog.com

[31] Kistler Instruments, Ltd. [Online]. Available: http://www.kistler.com/

[32] L. Ingber. (2004, Apr.) Adaptive Simulated Annealing for Matlab. [Online]. Available: http://www.igi.tugraz.at/lehre/MLA/WS01/ asamin.html

[33] J. Boland, H. Chao-Yuan, Y. Suzuki, and Y. C. Tai, "Micro electret power generator," in Proc. IEEE 16th Annu. Int. Conf. MEMS, Kyoto, Japan, Jan. 2003, pp. 538-541.

[34] E. M. Yeatman, "Advances in power sources for wireless sensor nodes," presented at the Int. Workshop on Wearable and Implantable Body Sensor Networks, London, U.K., Apr. 2004.

[35] D. C. Yates and A. S. Holmes, "Micro Power Radio Module," DC FET Project ORESTEIA Deliverable ND3.2, Tech. Rep., 2003. 


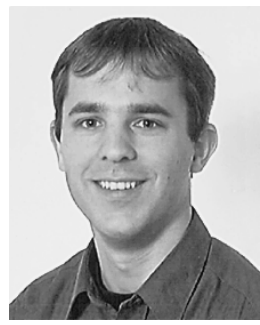

Thomas von Büren received the Dipl.-Ing. degree (M.Eng.) in electrical engineering from the Swiss Federal Institute of Technology (ETH) Zurich, Zurich, Switzerland, in 2000. He is currently pursuing the Ph.D. degree, focusing on micropower generators and their application in wearable computing.

He is currently a Research Assistant in the Wearable Computing Group, Department of Information Technology and Electrical Engineering, ETH Zurich.

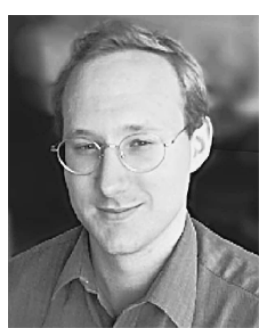

Paul D. Mitcheson received the M.Eng. degree in electrical and electronic engineering from Imperial College London, London, U.K., in 2001. He is currently pursuing the Ph.D. degree focusing on micropower generators and associated power electronics.

$\mathrm{He}$ is currently a Research Assistant in the Control and Power Research Group, Imperial College London.

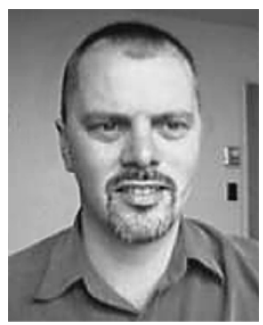

Tim C. Green received the B.Sc. (Eng.) degree in electrical engineering from Imperial College London, London, U.K., in 1986, and the Ph.D. degree in electrical engineering from Heriot-Watt University, Edinburgh, U.K., in 1990.

He was a Lecturer at Heriot-Watt University until 1994 and is now a Reader at Imperial College London and Deputy Head of the Control and Power Research Group. He has research interests in power electronics applied to generation and distribution of energy including issues of renewable and distributed generation, microgrids, power quality, active power filters, and flexible ac transmission systems.

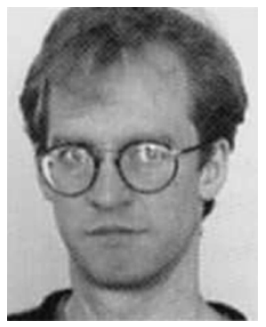

amplifiers.

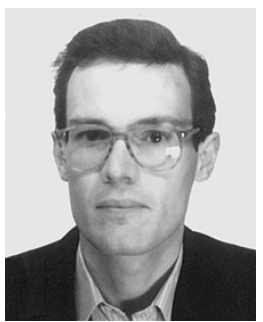

MEMS manufacture.
Eric M. Yeatman received the B.Sc. degree from Dalhousie University, Halifax, NS, Canada, in 1983, and the Ph.D. degree from Imperial College London, London, U.K., in 1989.

$\mathrm{He}$ is a member of staff in the Imperial College London's Electrical and Electronic Engineering Department, Optical and Semiconductor Devices Group, currently as Reader and Deputy Head of Group. His current research includes micromechanical actuators and generators, microstructures for microwave applications, and integrated optical

Andrew S. Holmes received the B.A. degree in natural sciences from Cambridge University, Cambridge, U.K., in 1987, and the Ph.D. degree in electrical engineering from Imperial College London, London, U.K., in 1992.

He is currently a Senior Lecturer in the Optical and Semiconductor Devices Group, Department of Electrical and Electronic Engineering, Imperial College London. His research interests are in the areas of micropower generation and conversion, MEMS devices for microwave applications, and laser processing for

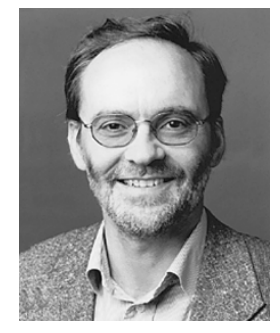

Gerhard Tröster received the M.Sc. degree in electrical engineering from the Technical University Karlsruhe, Karlsruhe, Germany, in 1978, and the $\mathrm{Ph} . \mathrm{D}$. degree in electrical engineering from the Technical University Darmstadt, Darmstadt, Germany, in 1984.

He is a Professor and Head of the Electronics Laboratory, Swiss Federal Institute of Technology (ETH) Zurich, Zurich, Switzerland. During the eight years he spent at Telefunken Corporation, Germany, he was responsible for various national and international research projects focused on key components for ISDN and digital mobile phones. He has authored and coauthored more than 100 articles and holds five patents. In 1997, he cofounded the spinoff u-blox ag. His field of research includes wearable computing, reconfigurable systems, signal processing, mechatronics, and electronic packaging. 